\title{
Multidrug-resistant Acinetobacter baumannii outbreaks: a global problem in healthcare settings
}

\author{
Mariana Neri Lucas Kurihara ${ }^{[1]}$, Romário Oliveira de Sales ${ }^{[1]}$, Késia Esther da Silva ${ }^{[1]}$, \\ Wirlaine Glauce Maciel ${ }^{[1]}$ and Simone Simionatto[1]
}

[1]. Universidade Federal da Grande Dourados, Laboratório de Pesquisa em Ciências da Saúde, Dourados, MS, Brasil.

\begin{abstract}
Introduction: The increase in the prevalence of multidrug-resistant Acinetobacter baumannii infections in hospital settings has rapidly emerged worldwide as a serious health problem. Methods: This review synthetizes the epidemiology of multidrug-resistant $A$. baumannii, highlighting resistance mechanisms. Conclusions: Understanding the genetic mechanisms of resistance as well as the associated risk factors is critical to develop and implement adequate measures to control and prevent acquisition of nosocomial infections, especially in an intensive care unit setting.
\end{abstract}

Keywords: Risk factors. Multidrug-resistant. ICU.

\section{METHODS}

A comprehensive search of the literature was performed using PubMed, ScienceDirect, and Web of Science. The search was restricted to original articles published in English related to risk factors, epidemiology, and multidrug-resistant $A$. baumannii (MDR- $A b$ ). The key words used were (Acinetobacter baumannii OR A. baumannii) AND infection AND (multidrug-resistant OR MDR) AND (ICU), or (Acinetobacter baumannii OR A. baumannii) AND risk factors AND epidemiology. Case reports or conference abstracts were excluded. Two independent investigators searched the electronic databases using an identical method. The full texts of articles were reviewed by two independent reviewers to determine whether they met the eligibility criteria for inclusion. References in the included articles were reviewed to explore additional papers.

\section{ACINETOBACTER BAUMANNII CONTEXT}

Acinetobacter spp. is a pathogen that belongs to the Moraxellaceae family, which consists of 59 different species ${ }^{1,2}$. In this family, Acinetobacter spp. is the fifth most frequently isolated microorganism, distributed across five continents, among the gram-negative bacteria involved in nosocomial infections ${ }^{3}$. It is known that the species Acinetobacter baumannii is an

\footnotetext{
Corresponding author: Dra. Simone Simionatto.

e-mail: simonesimionatto@ufgd.edu.br

(iD) https://orcid.org/0000-0003-2367-0915

Received 19 May 2020

Accepted 2 September 2020
}

opportunistic pathogen with clinical relevance ${ }^{3-6}$. The most frequent clinical manifestations are pneumonia associated with mechanical ventilation, bloodstream infections, urinary tract infections, and bacteremia associated with long periods of device use, meningitis, eye infections, intra-abdominal infections, surgical sites, the respiratory tract, and the gastrointestinal tract ${ }^{7,8}$. Nonetheless, this pathogen can survive in the intensive care unit (ICU) environment for up to four weeks due to its capacity to produce biofilms and thus contaminates patients admitted later ${ }^{9}$. Lipopolysaccharides (LPS), vesicles and proteins, polysaccharide capsules, phospholipases, proteases, outer membrane porins, and iron uptake systems are the most important factors for $A$. baumannii resistance ${ }^{10}$.

MDR- $A b$ is considered a hospital-acquired infection, which has been rapidly increasing worldwide due to the fitness effect of its resistance mutations ${ }^{3}$. The exacerbated and undue use of antibiotics associated with ineffective hospital interventions are related to the spread of MDR and consequently reduce treatment options. The World Health Organization (WHO) published in early 2017 a list of priorities for research into the development of active antibiotics against MDR and extensively resistant bacteria, which put $A$. baumannii first in the list of critical situations around the world ${ }^{11}$. It was estimated that multidrug-resistant $A$. baumannii can cost $\$ 33,510$ to $\$ 129,917$ per infection ${ }^{12}$. Moreover, patients with bacteremia can be related to high mortality rates due to multidrugresistant $A$. baumannii (56.2\%), when compared to A. baumannii strains with no multidrug resistance $(4.7 \%)^{13}$. An average of $10.6 \%$ of patients die as a result of infections caused by MDR- $A b^{12}$. 


\section{OVERVIEW OF A. BAUMANNII ANTIBIOTIC RESISTANCE}

The key resistance mechanisms of A. baumannii are the low permeability of the outer membrane, alteration in antibiotic binding sites, and mutations, which can cause upregulation or downregulation of efflux system activity ${ }^{4,10}$. Among these mechanisms, alteration of bacterial membrane permeability by the outer membrane proteins (OMPs) is associated with the loss or reduced expression of porins ${ }^{8}$. This group is represented by OmpA, OprD, and CarO proteins ${ }^{14}$. The OccD1 (OprD) channel of the Pseudomonas aeruginosa species plays an important role in the uptake of molecules such as imipenem and meropenem. This OM channel is closely related to the OM family in A. baumannii and is the largest pore described amongst Occ proteins with efficient in vitro uptake responsible for transporting small molecules, presenting a huge potential for future antibiotic design ${ }^{15}$.

The efflux system expels toxic compounds to the extracellular environment. Within it, five families of systems have been described in A. baumannii, such as the major facilitator super family (MFS), ATP binding cassette (ABC), resistance nodulation division (RND), small multidrug resistance family 1 (SMR), multidrug and toxic compound extrusion (MATE), and drug/metabolite transporter $(\mathrm{DMT})^{16}$. The RND family is well characterized and is represented by the AdeABC, AdeIJK, and AdeFGH efflux system ${ }^{17}$. Mutations can influence the expression of the efflux system, resulting in increased cases of clinical infections. A study highlighted resistance to aminoglicosides, tetracyclines, chloramphenicol, fluoroquinolones, some beta-lactams, and tigecycline related to mutations on the chromosome or plasmids ${ }^{18}$. The efflux systems CraA, AmvA/AedF, Tet(A), and Tet(B) of the MFS system are known to have a drug-specific substrate profile, and are involved in chloramphenicol, erythromycin, chlorhexidine, and tetracycline resistance ${ }^{19,20}$. The expression of Acel protein is strictly related to chlorhexidine transportation and the AbeM gene (a member of the MATE family), which confers resistance to fluoroquinolones through the $\mathrm{H}^{+}$antiport ${ }^{20,21}$. Quinolone resistance can be related to the $A b a Q$ gene, which belongs to the MFS transporter and has its $\mathrm{N}$ - and $\mathrm{C}$ - ends located in the cytoplasm, which confers its characteristic as a drug $\mathrm{H}^{+}$antiporter-1 (DHA1). AbaQ knockout in $A$. baumannii confirmed its involvement with quinolone susceptibility, resulting in decreased susceptibility caused by active efflux transportation ${ }^{22}$.

It is known that the fluoroquinolone resistance mechanism is mainly encoded by mutations in DNA gyrase ( $g y r A$, gyrB genes) and topoisomerase IV (parC, parE), with gyrB and parE mutated at a lower frequency. These mutations are sequential, as primary mutations in gyrA81 are followed by mutations in parC 88 and parC84 in A. baumannii. However, a study described strains carrying mutations in only the $\operatorname{par} C$ gene, revealing the involvement of other resistance mechanisms for fluoroquinolone ${ }^{23-24}$.

One of the main mechanisms of resistance to beta-lactam antibiotics is associated with changes in the structure or expression profile of penicillin binding proteins (PBPs) ${ }^{25}$. PBPs are transglycosylases, transpeptidases, and carboxypeptidases, enzymes located in the plasma membrane, and are involved in the synthesis of peptidoglycan, an essential component of the bacterial cell wall.
Once a PBP is acylated by a beta-lactam antibiotic, it is unable to catalyze hydrolysis of the covalent acyl-enzyme intermediate and is inactivated. Peptidoglycan transpeptidation cannot occur; thus, the cell wall is weakened ${ }^{25}$.

PBPs are divided into high molecular mass (HMM) and low molecular mass (LMM). The first is responsible for insertion into the cell wall, which, depending on the structure and catalytic activity of the $\mathrm{N}$-terminal domain, can be classified as class $\mathrm{A}$ or $\mathrm{B}^{26}$. Therefore, changes in PBP expression lead to decreased susceptibility to these antimicrobial agents, favoring the occurrence of beta-lactamresistant strain ${ }^{27}$. Due to the lack of interaction that occurs in the connection between beta-lactams and PBPs, the susceptibility of A. baumannii strains to beta-lactams has been observed ${ }^{27-29}$.

Mutations can occur and modify the binding of antibiotics, inactivating some lipids, such as lipid $\mathrm{A}^{30}$. Polymyxins interact with lipid A through the addition of phosphoethanolamine (PEtn), resulting in displacement of cations $\mathrm{Mg}^{2+}$ and $\mathrm{Ca}^{2+}$, which destabilizes the membrane. These molecules are mediated by the pmrCAB operon ${ }^{31-33}$. Alterations in the pmrA-pmrB two-component system, which is also involved in lipid A biosynthesis, upregulate pmrC, influencing the synthesis of PEtn. It is known that LPS is synthesized through the lpx pathway; mutations in $l p x A, l p x C$, and $\operatorname{lp} x D$ genes lead to deficiency in LPS production and its complete loss, conferring the colistin resistance phenotype ${ }^{34,35}$. Colistin resistance can be chromosomal or plasmid-encoded, carrying the mer gene ( $m c r-1$ to $m c r-5)^{36,37}$.

Carbapenemases, belonging to class A of Ambler (1980) and to group 2 of Bush and Jacob (2010) are considered one of the most versatile enzymatic families among $\beta$-lactamases, since they are able to hydrolyze most $\beta$-lactam antibiotics, such as carbapenems, penicillins, cephalosporins, and monobactams, in addition to being resistant against some commercial $\beta$-lactamase inhibitors ${ }^{35,38}$. Enzymes such as KPC-2, KPC-3, KPC-4, and KPC-10 $0^{39}$, as well as GES-11, GES-12, and GES-14 ${ }^{40}$, have already been described in $A$. baumannii ${ }^{38}$.

Metallo- $\beta$-lactamases belong to class B of Ambler (1980) and group 3 of Bush and Jacoby (2010). They confer resistance against penicillins, cephalosporins, and carbapenems, and are inhibited by $\beta$-lactamase inhibitors (clavulanic acid, sulbactam, and tazobactam). The enzymes representing this family are VIM-1 and NDM-1, commonly related to penicillin hydrolysis ${ }^{39-43}$. Class C of Ambler (1980), group 1 of Bush and Jacob (2010), is represented by chromosomal cephalosporinases $(\mathrm{AmpC})$, which hydrolyze penicillins, and cephalosporins at a low level. When the insertion element ISAbal or ISAba125 is inserted upstream of the $b l a_{\text {AmpC }}$ gene, it is overexpressed, resulting in resistance to extended-spectrum cephalosporins as upstream ISAba induces strong promoter sequences ${ }^{44,45}$.

Oxacillinases belong to class D of Ambler (1980) and group 2 of Bush and Jacob (2010) and are encoded by the $b l a_{\text {OXA }}$ genes. These proteins hydrolyze carbapenems and penicillins at a low level and has weak hydrolysis of second and third generation cephalosporins ${ }^{44}$. Oxacillinases have been reported in clinical isolates of $A$. baumannii associated with hospital outbreaks ${ }^{46}$. Six subgroups of Class D carbapenem-hydrolyzing enzymes (CHDLs), including OXA-23, 
OXA-24, OXA-51, OXA-58, OXA-143, and OXA-235, were identified $^{47}$. These enzymatic groups hydrolyze penicillins at a high level and carbapenems at a low level. However, the presence of insertion sequence (IS) is considered a strong promoter for the increase of oxacillin expression and dissemination ${ }^{48}$. It was reported that the ISAbal/bla ${ }_{\text {OXA-23 }}$ or ISAbal/bla $a_{\text {OХA-51 }}$ combination amplified resistance to carbapenems ${ }^{49}$.

Aminoglycosides bind to $16 \mathrm{~S}$ rRNA in the $30 \mathrm{~S}$ ribosomal subunits and inhibit protein synthesis. Resistance is mediated by aminoglycoside-modifying enzymes (AMEs), such as acetyltransferases (AAC), adenyltransferases (ANT), and phosphotransferases (APH), which are found on mobile elements such as transposons and plasmids. AAC enzymes are responsible for modifying amino groups, while the ANT and APH enzymes act on hydroxyl groups, breaking bonds and inactivating the antibiotic molecule ${ }^{10}$. Methylase production (armA, rmtA, rmtB, $r m t C, r m t D$ ) decreases the affinity of the aminoglycosides for $30 \mathrm{~S}$ ribosomal subunits ${ }^{50}$. A study with carbapenem-resistant (CR) A. baumannii identified $97.2 \%$ of the isolates carrying the aph(3')$V I$ gene, with the majority found in 4 different clusters (A, B, C, and $\mathrm{E}$ ), conferring resistance to amikacin, and group $\mathrm{D}$, harboring AME genes ( $a a c\left(6^{\prime}\right)-I b, a a c(3)-I a$, and $\left.a p h\left(3^{\prime}\right)-I a\right)$, responsible for gentamicin resistance and intermediate resistance to amikacin ${ }^{51,52}$. The presence of methylase armA coexisting with $b l a_{\text {OXA-23 }}$ in MDR $A$. baumannii has been previously described and identified in quinolone-resistant $A$. baumannii ${ }^{53,54}$.

In addition to the multiple mechanisms of resistance, A. baumannii can acquire resistance genes through mobile genetic elements. Mobile elements, such as IS, transposons, genomic islands, integrons, and plasmids, are related to variations in the insertion site and carry strong transcriptional promoters that are abundantly synthesized ${ }^{55,56}$. Multiple $A$. baumannii plasmids have been reported: pA297-1, carrying gentamicin, kanamycin, and tobramycin resistance genes; pA297-3, carrying sulfonamide and streptomycin resistance genes; and pAb-G7-2, carrying an amikacin resistance gene ${ }^{57,58}$.

Transposons, such as Tn2006, $\operatorname{Tn} 2007$, and $\operatorname{Tn} 2008$, increase the spread of resistance genes and may present integrons, which were captured and express exogenous resistance genes ${ }^{40,48,59}$. Thus, integrons are composed of gene cassettes, and classes 1 and 2 are commonly found in A. baumannii clinical isolates ${ }^{60-62}$. As previously stated, insertion sequences act as strong promoters that increase the resistance levels of OXA carbapenemases in A. baumannii isolates ${ }^{47,59,63}$. Insertion sequence Acinetobacter baumannii (ISAba) can be located upstream of the resistant gene, overexpressing genes such as $A m p C$ and OXA-51, which increases cephalosporin resistance ${ }^{64,65}$. Resistance to colistin in $A$. baumannii clinical isolates was related to the presence of the ISAba125 at the 3' end of the hns gene, disrupting the normal expression of a transcriptional gene regulator ${ }^{66}$.

\section{RISK FACTORS RELATED TO A. BAUMANNII}

Risk factors are directly related to increased susceptibility in hospitalized patients who develop some type of infectious disease involving bacterial resistance, consequently resulting in mortality in nosocomial environments. Investigation of the risk factors associated with $A$. baumannii infection/colonization contributes to the prevention and control of bacterial resistance, reducing the impact of $A$. baumannii isolates ${ }^{67}$ (Table 1 and Table 2). The prevalence of $A$. baumannii infection and colonization is higher in ICUs, since patients with severe clinical conditions are hospitalized in such wards. In addition, these patients have compromised immune systems due to the presence of comorbidities, altered nutritional status, prolonged hospitalization, invasive procedures, immunosuppressive drugs, and broad-spectrum antibiotics ${ }^{67,68}$.

Skin colonization, length of hospital stays $>7$ days, use of corticosteroids, and invasive procedures such as central venous catheter or tracheostomy, were the main risk factors related to the development of pneumonia associated with mechanical ventilation by MDR A. baumannii in hospitalized patients (Table 1) ${ }^{69,70}$. Risk factors such as use of urinary catheters for more than 6 days, ICU contact pressure $>4$ days, presence of gastrectomy tubes, chemotherapy, organ transplantation, chronic diseases, invasive procedures, recent bacteremia, tumors, hematological diseases, recurrent hospitalizations, hospitalization time $>7$ days, transfer from another hospital, and previous use of carbapenems or broadspectrum cephalosporins were related to acquisition of MDR A. baumannii infection in adult patients hospitalized in the $\mathrm{ICU}^{69,71}$. Isolation of MDR A. baumannii after medical ICU (MICU) admission was related to a greater likelihood of the patient being older $^{72}$. Previous hospitalization was associated with the isolation of A. baumannii after admission to the surgical ICU (SICU). Positive colonization in SICU was strongly correlated with heart failure, paralysis, human immunodeficiency virus infection and acquired immune deficiency syndrome (HIV-AIDS), and rheumatoid arthritis $^{73}$.

Bloodstream infections by $A$. baumannii are frequent in ICUs and have been associated with central venous catheters, mechanical ventilation, pneumonia, drain use, and respiratory and cardiovascular failure ${ }^{74}$. The risk of bacteremia caused by A. baumannii was associated with respiratory failure, mechanical ventilation, endotracheal tubes, central venous catheters, surgical procedures, and previous use of antibiotics ${ }^{75,76}$.

Newborns are considered susceptible to A. baumannii colonization and infections, since they have immature immune systems. The risk is greater for newborns if they are also preterm $(<28$ weeks $)$ and underweight $(<2,500 \mathrm{~g})^{76,77}$. Birth weight $<2500$ grams, respiratory syndromes, parental feeding, re-intubation, carbapenem use, mechanical ventilation, hematologic diseases, neutropenia $>3$ days, previous use of broad-spectrum antibiotics, use of invasive devices, immunosuppressants, corticosteroids, previous hospitalization, and ICU stay $>3$ days were considered risk factors for the acquisition of $A$. baumannii infections in the neonatal ICU (Table 2) ) $^{78-80}$.

Bloodstream infections caused by A. baumannii in neonates were related to the use of mechanical ventilation, and additionally to the presence of traumatic brain injury, previous use of antibiotics, hospitalization $>7$ days, and use of mechanical ventilation $>7$ days $^{81-83}$. The weight of newborns (1000-1499 g), previous use of cephalosporins, surfactant replacement therapy, re-intubation, and umbilical artery catheterization were also indicated as risk factors 
TABLE 1: Risk factors associated with infection and colonization caused by $A$. baumannii in adult ICUs.

\begin{tabular}{|c|c|c|c|c|c|c|c|}
\hline Study & $\begin{array}{l}\text { Place of } \\
\text { Study }\end{array}$ & Study Period & $\begin{array}{l}\text { No. of } \\
\text { Patients }\end{array}$ & Cases & Controls & Risk Factors & P-value \\
\hline JANG et al., 2009 & Taiwan & $1997-2006$ & 154 & $\begin{array}{l}77 \text { patients } \\
\text { with } A B \\
\text { bloodstream } \\
\text { infection. }\end{array}$ & $\begin{array}{l}77 \text { patients with } \\
\text { bloodstream infection } \\
\text { without } A B .\end{array}$ & $\begin{array}{l}\text { Use of central venous catheter, } \\
\text { mechanical ventilation, } \\
\text { colonization by } A B \text {, respiratory } \\
\text { failure, cardiovascular failure. }\end{array}$ & $P<0.05$ \\
\hline YE et al., 2010 & Germany & $2001-2005$ & 209 & $\begin{array}{l}49 \text { patients } \\
\text { with MDRAB. }\end{array}$ & $\begin{array}{l}160 \text { patients with } \\
\text { CSAB. }\end{array}$ & $\begin{array}{l}\text { Previous use of antibiotics, use } \\
\text { of mechanical ventilation, }>60 \\
\text { years, length of hospital stay. }\end{array}$ & $P<0.05$ \\
\hline $\begin{array}{l}\text { ROCHA et al., } \\
2008\end{array}$ & Brazil & 2005-2006 & 275 & $\begin{array}{l}84 \text { patients } \\
\text { with PAVM. }\end{array}$ & $\begin{array}{l}191 \text { patients without } \\
\text { PAVM. }\end{array}$ & $\begin{array}{l}\text { Stay }>7 \text { days in hospital, use of } \\
\text { corticoids, invasive procedures, } \\
\text { use of central venous catheter, } \\
\text { and tracheostomy. }\end{array}$ & $P<0.05$ \\
\hline $\begin{array}{l}\text { BROTFAIN et al, } \\
2016\end{array}$ & Israel & $2005-2011$ & 129 & $\begin{array}{l}46 \text { patients } \\
\text { with } \\
\text { pneumonia } \\
\text { and positive } \\
\text { sputum culture } \\
\text { for MDRAB } \\
72 \mathrm{~h} \text { after MV } \\
\text { onset and } \\
\text { bacteremia. }\end{array}$ & $\begin{array}{l}83 \text { patients with } \\
\text { pneumonia and } \\
\text { positive sputum } \\
\text { culture for MDRAB } \\
72 \mathrm{~h} \text { after the } \\
\text { onset of MV, } \\
\text { without developing } \\
\text { bacteremia. }\end{array}$ & $\begin{array}{l}\text { Hospitalization }>3 \text { days in the } \\
\text { ICU, advanced age, and recent } \\
\text { bacteremia. }\end{array}$ & $P<0.05$ \\
\hline $\begin{array}{l}\text { BLANCO et al., } \\
2017\end{array}$ & $\begin{array}{l}\text { United } \\
\text { States }\end{array}$ & 2005-2009 & 101 & $\begin{array}{l}90 \text { patients } \\
\text { with MDRAB. }\end{array}$ & $\begin{array}{l}11 \text { patients with } \\
\text { CSAB. }\end{array}$ & $\begin{array}{l}\text { Advanced age, previous } \\
\text { hospitalization, heart failure, } \\
\text { paralysis, HIV-AIDS, and } \\
\text { rheumatoid arthritis. }\end{array}$ & $P<0.05$ \\
\hline ELLIS et al., 2015 & $\begin{array}{l}\text { United } \\
\text { States }\end{array}$ & 2006-2012 & 671 & $\begin{array}{l}302 \text { patients } \\
\text { with infection } \\
\text { caused by } \\
\text { MDRAB. }\end{array}$ & $\begin{array}{l}369 \text { patients with } \\
\text { infection caused by } \\
\text { CSAB. }\end{array}$ & $\begin{array}{l}\text { Length of hospital stay, transfer } \\
\text { from another hospital, previous } \\
\text { use of antibiotics }\end{array}$ & $P<0.25$ \\
\hline $\begin{array}{l}\text { HENIG et al., } \\
2015\end{array}$ & Israel & $2007-2012$ & 2380 & $\begin{array}{l}1190 \text { patients } \\
\text { with CRAB. }\end{array}$ & $\begin{array}{l}1190 \text { patients without } \\
A B \text {. }\end{array}$ & $\begin{array}{l}\text { Chemotherapy, organ } \\
\text { transplant, chronic diseases, } \\
\text { invasive procedures, } \\
\text { recent bacteremia, tumor, } \\
\text { hematological diseases, and } \\
\text { recurrent hospitalizations. }\end{array}$ & $P<0.05$ \\
\hline JUNG et al., 2010 & $\begin{array}{l}\text { South } \\
\text { Korea }\end{array}$ & $2008-2009$ & 200 & $\begin{array}{l}108 \text { patients } \\
\text { with } \\
\text { bacteremia } \\
\text { caused by } A B \text {. }\end{array}$ & $\begin{array}{l}92 \text { patients without } \\
\text { bacteremia. }\end{array}$ & $\begin{array}{l}\text { Respiratory failure, mechanical } \\
\text { ventilation, tracheal tube, } \\
\text { central venous catheter, } \\
\text { bacteremia caused by other } \\
\text { microorganisms, previous use } \\
\text { of antibiotics. }\end{array}$ & $P<0.05$ \\
\hline $\begin{array}{l}\text { NUTMAN et al., } \\
2014\end{array}$ & Israel & $2008-2011$ & 172 & $\begin{array}{l}83 \text { patients } \\
\text { with } \\
\text { bacteremia } \\
\text { who died } \\
\text { within } 14 \text { days. }\end{array}$ & $\begin{array}{l}89 \text { patients with } \\
\text { bacteremia who } \\
\text { survived after } 14 \\
\text { days. }\end{array}$ & $\begin{array}{l}\text { Disease severity and surgical } \\
\text { procedure. }\end{array}$ & $P \leq 0.10$ \\
\hline $\begin{array}{l}\text { CHUSRI et al., } \\
2015\end{array}$ & Thailand & $2010-2011$ & 394 & $\begin{array}{l}139 \text { patients } \\
\text { with CRAB. }\end{array}$ & $\begin{array}{l}197 \text { patients without } \\
A B \text { and } 58 \text { patients } \\
\text { with CSAB. }\end{array}$ & $\begin{array}{l}\text { Use of fluoroquinolones, broad } \\
\text { spectrum cephalosporins, and } \\
\text { carbapenems }>3 \text { days. }\end{array}$ & $P<0.05$ \\
\hline $\begin{array}{l}\text { MOGHNIEH et al., } \\
2016\end{array}$ & Lebanon & $2012-2013$ & 257 & $\begin{array}{l}40 \text { patients } \\
\text { with } A B \text {. }\end{array}$ & $\begin{array}{l}217 \text { patients without } \\
A B \text {. }\end{array}$ & $\begin{array}{l}\text { Use of urinary catheter, ICU } \\
\text { contact pressure, gastrectomy } \\
\text { tube, and carbapenem use. }\end{array}$ & $P<0.05$ \\
\hline GUO et al., 2016 & China & $2012-2015$ & 87 & $\begin{array}{l}64 \text { patients } \\
\text { with } \\
\text { bloodstream } \\
\text { infection by } \\
\text { MDRAB. }\end{array}$ & $\begin{array}{l}23 \text { patients with } \\
\text { bloodstream infection } \\
\text { by CSAB. }\end{array}$ & $\begin{array}{l}\text { Pneumonia, drain use, ICU } \\
\text { stay }>7 \text { days, and use of } \\
\text { mechanical ventilation. }\end{array}$ & $P<0.05$ \\
\hline
\end{tabular}

AB: A. baumannii; MDRAB: multidrug-resistant A. baumannii; PAVM: pneumonia associated with mechanical ventilation; MV: mechanical ventilation; CRAB: carbapenem-resistant $A$. baumannii; CSAB: carbapenem-susceptible $A$. baumannii; ICU: intensive care unit. 
TABLE 2: Risk factors associated with infection and colonization caused by $A$. baumannii in pediatric and neonatal ICUs.

\begin{tabular}{|c|c|c|c|c|c|c|c|}
\hline Study & $\begin{array}{l}\text { Place of } \\
\text { study }\end{array}$ & $\begin{array}{l}\text { Study } \\
\text { period }\end{array}$ & $\begin{array}{l}\text { No. of } \\
\text { patients }\end{array}$ & Cases & Controls & Risk factors & P-value \\
\hline BRITO et al., 2010 & Brazil & 2001-2002 & 33 & $\begin{array}{l}11 \text { patients with } \\
\text { infectious conditions } \\
\text { caused by } A B \text {. }\end{array}$ & $\begin{array}{l}22 \text { patients without } \\
\text { infectious conditions } \\
\text { caused by } A B \text {. }\end{array}$ & $\begin{array}{l}\text { Birth weight }<2500 \\
\text { grams, respiratory } \\
\text { syndromes, parental } \\
\text { feeding, re-intubation, } \\
\text { carbapenem use, and } \\
\text { mechanical ventilation. }\end{array}$ & $P<0.05$ \\
\hline DENG et al., 2011 & China & $2002-2008$ & 349 & $\begin{array}{l}117 \text { patients with PAVM } \\
\text { caused by } A B \text {. }\end{array}$ & $\begin{array}{l}232 \text { patients without } \\
\text { PAVM caused by } A B \text {. }\end{array}$ & $\begin{array}{l}\text { Use of mechanical } \\
\text { ventilation> } 7 \text { days. }\end{array}$ & $P<0.01$ \\
\hline HSU et al., 2014 & Taiwan & $2004-2010$ & 248 & $\begin{array}{l}37 \text { patients with } \\
\text { bacteremia caused } \\
\text { by } A B .\end{array}$ & $\begin{array}{l}74 \text { patients without } \\
\text { bacteremia and } 137 \\
\text { patients with bacteremia } \\
\text { caused by Escherichia } \\
\text { coli or Klebsiella spp. }\end{array}$ & $\begin{array}{l}\text { Cholestasis, gestational } \\
\text { age }<29 \text { weeks. }\end{array}$ & $P<0.05$ \\
\hline LEE et al., 2017 & China & 2004-2014 & 40 & $\begin{array}{l}37 \text { patients with } A B \\
\text { susceptible to imipenem }\end{array}$ & $\begin{array}{l}3 \text { patients with } A B \\
\text { resistant to imipenem }\end{array}$ & $\begin{array}{l}\text { Prematurity, low birth } \\
\text { weight }(70 \%<1500 \mathrm{~g}) \text {, } \\
\text { prolonged intubation, } \\
\text { percutaneous use of } \\
\text { central venous catheter, } \\
\text { inappropriate initial } \\
\text { therapy, infection within } \\
\text { the first } 10 \text { days of life, } \\
\text { use of imipenem for } \\
\text { up to } 5 \text { days, and high } \\
\text { frequency oscillation } \\
\text { ventilation. }\end{array}$ & $P<0.05$ \\
\hline $\begin{array}{l}\text { PUNPANICH et al., } \\
2012\end{array}$ & Thailand & $2005-2010$ & 176 & $\begin{array}{l}91 \text { patients with } \\
\text { bacteremia caused by } \\
\text { CRAB. }\end{array}$ & $\begin{array}{l}85 \text { patients with } \\
\text { bacteremia caused by } \\
\text { CSAB. }\end{array}$ & $\begin{array}{l}\text { Prematurity, use of } \\
\text { mechanical ventilation, } \\
\text { previous exposure to } \\
\text { carbapenems. }\end{array}$ & $P<0.05$ \\
\hline $\begin{array}{l}\text { HOSOGLU et al., } \\
2012\end{array}$ & Turkey & $2006-2007$ & 192 & $\begin{array}{l}64 \text { patients with } A B \\
\text { sepsis. }\end{array}$ & $\begin{array}{l}128 \text { patients with blood } \\
\text { samples without } A B \text {. }\end{array}$ & $\begin{array}{l}\text { Stay in the ICU> } 7 \text { days, } \\
\text { re-intubation. }\end{array}$ & $P<0.001$ \\
\hline $\begin{array}{l}\text { De OLIVEIRA } \\
\text { COSTA et al., } 2015\end{array}$ & Brazil & 2009-2012 & 101 & $\begin{array}{l}47 \text { patients with } \\
\text { infection caused by } \\
\text { BGN. }\end{array}$ & $\begin{array}{l}54 \text { patients without } \\
\text { infection caused by BGN. }\end{array}$ & $\begin{array}{l}\text { Hematologic diseases, } \\
\text { neutropenia }>3 \\
\text { days, previous use of } \\
\text { antibiotics, previous } \\
\text { hospitalization, stay in } \\
\text { the ICU }>3 \text { days. }\end{array}$ & $P<0.05$ \\
\hline $\begin{array}{l}\text { THATRIMONTRICHAI } \\
\text { et al., } 2013\end{array}$ & Thailand & $2009-2014$ & 101 & $\begin{array}{l}63 \text { patients with } C R A B \\
\text { pneumonia and } 13 \\
\text { patients with CSAB. }\end{array}$ & $\begin{array}{l}25 \text { patients with } \\
\text { pneumonia without } \\
\text { bacterial growth or } \\
\text { caused by other } \\
\text { microorganisms. }\end{array}$ & $\begin{array}{l}\text { Weight of newborns, } \\
\text { previous use of } \\
\text { cephalosporins, } \\
\text { surfactant replacement } \\
\text { therapy, re-intubation, } \\
\text { umbilical artery } \\
\text { catheterization. }\end{array}$ & $P<0.05$ \\
\hline REDDY et al., 2015 & $\begin{array}{l}\text { South } \\
\text { Africa }\end{array}$ & 2010 & 388 & $\begin{array}{l}194 \text { patients with blood } \\
\text { culture or respiratory } \\
\text { sample positive for } A B \text {. }\end{array}$ & $\begin{array}{l}194 \text { patients with blood } \\
\text { culture or negative } \\
\text { respiratory sample for } \\
A B \text {. }\end{array}$ & $\begin{array}{l}\text { Mechanical ventilation } \\
\text { and traumatic brain } \\
\text { injury. }\end{array}$ & $P<0.05$ \\
\hline ZARRILLI et al., 2012 & Italy & $2010-2011$ & 161 & 22 patients with $A B$. & $\begin{array}{l}139 \text { patients without } A B \\
\text { in the first } 48 \mathrm{~h} .\end{array}$ & $\begin{array}{l}\text { Use of mechanical } \\
\text { ventilation and central } \\
\text { venous catheter. }\end{array}$ & \\
\hline TRAN et al., 2015 & Vietnam & $2010-2011$ & 2555 & $\begin{array}{l}69 \text { patients with sepsis } \\
\text { caused by } A B \text {. }\end{array}$ & $\begin{array}{l}2486 \text { patients without } \\
\text { sepsis caused by } A B .\end{array}$ & $\begin{array}{l}\text { Maternal infection, } \\
\text { gestational age, central } \\
\text { catheter, surgical } \\
\text { procedure, and blood } \\
\text { transfusion. }\end{array}$ & $P<0.05$ \\
\hline KUMAR et al., 2014 & India & 2010-2012 & 65 & $\begin{array}{l}33 \text { patients with CRAB } \\
\text { bloodstream infection. }\end{array}$ & $\begin{array}{l}32 \text { patients without CSAB } \\
\text { bloodstream infection. }\end{array}$ & $\begin{array}{l}\text { Previous use } \\
\text { of antibiotics, } \\
\text { hospitalization }>7 \text { days, } \\
\text { use of mechanical } \\
\text { ventilation }>7 \text { days. }\end{array}$ & $P<0.05$ \\
\hline WEl et al., 2014 & Taiwan & $2010-2013$ & 59 & $\begin{array}{l}12 \text { deaths due to sepsis } \\
\text { caused by MDRAB. }\end{array}$ & $\begin{array}{l}47 \text { deaths due to } \\
\text { sepsis caused by other } \\
\text { microorganisms. }\end{array}$ & $\begin{array}{l}\text { Prolonged intubation, } \\
\text { mechanical ventilation, } \\
\text { peripheral central } \\
\text { venous catheter, } \\
\text { umbilical catheter, } \\
\text { total parental nutrition, } \\
\text { ICU stay > } 7 \text { days, } \\
\text { surgical procedure, } \\
\text { and bronchopulmonary } \\
\text { dysplasia. }\end{array}$ & $P<0.05$ \\
\hline MACIEL et al., 2017 & Brazil & 2013-2015 & 21 & $\begin{array}{l}21 \text { patients with } A B \\
\text { colonization without } \\
\text { clinical manifestation. }\end{array}$ & $\begin{array}{l}17 \text { patients without } \\
\text { sepsis. }\end{array}$ & $\begin{array}{l}\text { Low birth weight, } \\
\text { prematurity, } \\
\text { hospitalization time, } \\
\text { previous exposure to } \\
\text { beta-lactams, use of } \\
\text { peripheral access, and } \\
\text { respiratory syndromes. }\end{array}$ & $P<0.05$ \\
\hline
\end{tabular}

AB: A. baumannii; PAVM: pneumonia associated with mechanical ventilation; CRAB: carbapenem-resistant $A$. baumannii; CSAB: carbapenem-susceptible $A$. baumannii; BGN: gram-negative bacillus; MDRAB: multidrug-resistant $A$. baumannii; ICU: intensive care unit. 
TABLE 3: Outbreaks of Acinetobacter baumannii in Brazil.

\begin{tabular}{|c|c|c|c|c|c|c|}
\hline Study & $\begin{array}{l}\text { Place of } \\
\text { study }\end{array}$ & $\begin{array}{c}\text { Year of } \\
\text { outbreak }\end{array}$ & $\begin{array}{l}\text { Place of } \\
\text { outbreak }\end{array}$ & $\begin{array}{c}\text { No. of } \\
\text { patients }\end{array}$ & Antibiotic Resistance & Reported genes \\
\hline $\begin{array}{l}\text { DALIA-COSTA et } \\
\text { al., } 2003\end{array}$ & Curitiba & 1999 & Ward & 8 & IPM, MEM, CIP, and AMG & $b / a_{\text {OXA-23 }}$ \\
\hline $\begin{array}{l}\text { BRITO et al., } \\
2005\end{array}$ & Uberlândia & 2005 & $\mathrm{NICU}$ & 11 & GEN, CIP, CAZ, FEP, and ATM & - \\
\hline $\begin{array}{l}\text { TAKAGI et al., } \\
2009\end{array}$ & São Paulo & $2005-2006$ & $\mathrm{ICU}$ & 8 & $\begin{array}{l}\text { PIP, TZP, CAZ, CTX, ATM, IPM, MEM, CIP, } \\
\text { AMK, GEN, and SXT }\end{array}$ & $b / a_{\text {OXA-51 }}$ \\
\hline $\begin{array}{l}\text { MARTINS et al., } \\
2009\end{array}$ & Porto Alegre & 2007 & DHW & 53 & CIP, GEN, TZP, and SXT & $b / a_{\mathrm{OXA}-51,} b / a_{\mathrm{OXA}-23}$ \\
\hline $\begin{array}{l}\text { GUSATTI et al., } \\
2012\end{array}$ & Porto Alegre & 2007 & Ward & 74 & $\begin{array}{l}\text { IPM, MEM, AMK, CIP, GEN, CET, AMA, SXT, } \\
\text { and TIM }\end{array}$ & $\begin{array}{l}b / a_{\text {OXA-51 }} \text { bla } \\
\text { ISAXA-5a1/OXA-51 } \\
\text { OXA-65, }\end{array}$ \\
\hline $\begin{array}{l}\text { PAGANO et al., } \\
2015\end{array}$ & Porto Alegre & 2011 & DHW & 122 & FEP, CIP, CAZ, AMA, AMK, PMB, IMP and MEM & bla $a_{\text {OXA-23 }}$ \\
\hline $\begin{array}{l}\text { CASTILHO et al., } \\
2017\end{array}$ & Goiás & 2010 & $\mathrm{ICU}$ & 64 & AMA, FEP, AMK, PMB, and TGC & $\begin{array}{c}\text { ISAba1/OXA-23 and } \\
\text { ISAba1/OXA-51, bla } \text { OXA-51, }, \\
\text { bla }_{\text {OXA-23, }} \text { bla } a_{\text {OXA-58 }}\end{array}$ \\
\hline $\begin{array}{l}\text { MACIEL et al., } \\
2017\end{array}$ & Dourados & 2013-2015 & $\mathrm{NICU}$ & 21 & $\begin{array}{c}\text { AMA, TZP, CAZ, CRO, FEP, GEN, AMK, CIP, } \\
\text { and TGC. }\end{array}$ & $\begin{array}{l}\text { ISAba1/OXA-23 and } \\
\text { ISAba1/OXA-51 }\end{array}$ \\
\hline
\end{tabular}

ICU: intensive care unit; NICU: neonatal intensive care unit; DHW: different hospital wards; IPM: imipenem; MEM: meropenem; CIP: ciprofloxacin; AMG: aminoglycoside; GEN: gentamicin; CAZ: ceftazidime; FEP: cefepime; ATM: aztreonam; TZP: piperacillin/tazobactam; AMK: amikacin; SXT: trimethoprim/sulfamethoxazole; CET: cephalothin; TIM: ticarcillin/clavulanic acid; PMB: polymyxin B; TGC: tigecycline; CRO: ceftriaxone; AMA: ampicillin/sulbactam; CTX: cefotaxime; PIP: piperacillin.

for the development of neonatal pneumonia caused by carbapenemresistant $A$. baumannii ${ }^{84}$. Maternal infection, gestational age among 26 to 36 weeks, use of central venous catheters, surgical procedures, blood transfusions, prolonged intubation, use of mechanical ventilation, central peripheral venous catheters, umbilical catheters, total parental nutrition, ICU stay $>7$ days, surgical procedures, and bronchopulmonary dysplasia were described as risk factors for sepsis by $A$. baumannii ${ }^{77,85}$. Cholestasis, gestational age $<29$ weeks, prematurity, low birth weight $(70 \%<1500 \mathrm{~g})$, prolonged intubation, central venous catheters, use of imipenem for up to 5 days, mechanical ventilation, and prior carbapenem exposure are related to A. baumannii bacteremia in neonates ${ }^{10,86,87}$. Similar results were reported for colonization in neonates ${ }^{88}$. These studies pinpoint persistent endemic isolates in hospitals, highlighting the need to implement efficient control measures and prevent outbreaks.

Seasonality of A. baumannii infection is another risk factor that should be taken into consideration. A systematic review compiled studies showing $57.1 \%(12 / 21)$ of $A$. baumannii infections occurred in warmer seasons. The hypothesis for this was that it was due to enhanced lipid A moiety regulation, which was responsible for the virulence; it was also reported there was biofilm formation and a higher flow of people entering the hospital facility (carriers, patients, healthcare workers, and sanitation workers) in warmer months. This study highlights the importance of correlating different factors of A. baumannii adaptability in the ambient environment to implement preventive measures for seasonal peaks of infection ${ }^{89}$.

Information related to colonization pressure (CP) is important for mediating risk factors. $\mathrm{CP}$ is a tool to measure the proportion of $A$. baumannii reservoirs within a health care facility. For A. baumannii surveillance, $\mathrm{CP}$ can help enhance patient screening and determine infection control measures ${ }^{90,91}$.

\section{MOLECULAR EPIDEMIOLOGY OF A. BAUMANNII IN BRAZIL}

In Brazil, the first outbreak associated with OXA-23-producing A. baumannii isolates was in $1999^{92}$. Subsequently, different outbreaks were reported ${ }^{93}$. A. baumannii dissemination in different Brazilian hospitals was associated with $b l a_{\text {OXA-51 }}$ and $b l a_{\text {OXA-23 }}$ genes and highlighted the prevalence of ISAbaI/OXA-23 and ISAbaI/ OXA-51 genetic profiles ${ }^{94}$. Isolates carrying the $b l a_{\text {OXA-51 }}, b l a_{\text {OXA-58, }}$, and $b l a_{\mathrm{OXA}-23}$ genes, and ISAbal upstream of OXA-51 and OXA23 were found in different ICUs, indicating an outbreak of crosscontamination among patients, equipment, or medical staff ${ }^{94}$. The $b l a_{\text {OXA-58 }}$ and $b l a_{\text {OXA-65 }}$ genes with the upstream ISAbal sequence for both genes have been reported. The bla ${ }_{\text {OXA-58 }}$ gene is prevalent in Argentina, indicating a possible spread from the border with Rio Grande do Sul ${ }^{95}$. In addition, two genotypes of OXA-23-producing A. baumannii were present at 8 hospitals in the same city, suggesting the spread of isolates in these environments ${ }^{93}$. The sequence type (ST) 156, ST25, and ST160 were identified in a Brazilian hospital ${ }^{96}$. Cephalosporin-resistant A. baumannii and producers of extendedspectrum beta-lactamases (ESBL) were identified in a neonatal intensive care unit (NICU), causing septicemia in hospitalized neonates (Table 3) ${ }^{5}$. A study in neonates described most isolates as belonging to ST1 and had ISAbal upstream of the $b l a_{\text {OXA-51 }}$ and $b l a_{\text {OXA-23 }}$ genes $^{88}$.

A study in Recife, Brazil described isolates belonging to ST1, ST15, ST25, ST79, ST113, and ST881 (related to ST1). Among them, ST79 and ST113 were found to be more virulent and presented resistance genes. ST113 and ST15 were commonly found in all 5 hospitals of the study, while ST79 was found in 4 hospitals and ST1 in 3 hospitals. Among the CCs circulating between hospitals, Leal et al. described $\mathrm{CC} 1, \mathrm{CC} 15$, and $\mathrm{CC} 113$, which are globally 
spread types, and CC79, which is found in South America, North America, and Europe ${ }^{97}$.

A study carried out in nine hospitals in South America identified A. baumannii clinical isolates presenting bla $a_{\text {OXA-51 }}$, bla $a_{\text {OXA-23, }}$, bla $a_{\text {OXA-72 }}, b l a_{\text {OXA-132 }}, b l a_{\text {OXA-65 }}, b l a_{\text {OXA-69 }}$, and bla $a_{\text {OXA-64 }}$ genes. Multilocus sequence type (MLST) analysis identified ST79, ST25, and $\mathrm{ST}_{15}{ }^{98}$. The two major clonal complexes (CC) found in bla ${ }_{\mathrm{OXA}-23}$ multidrug-resistant A. baumannii are CC15 and CC79, and CC15 has already been described in 9 Brazilian states ${ }^{77}$. In addition, ST15 was described in other countries, such as Argentina and Turkey, and ST79 was described in the United States, Canada, and Spain ${ }^{99}$. Of the clonal profiles identified, ST15 and ST79 were described in several countries, indicating their spread among hospitals around the world and high mortality rates ${ }^{100}$.

The Antimicrobial Surveillance Program (SENTRY) evaluated the prevalence of Acinetobacter spp. and other gram-negative bacilli isolated from Latin American (Argentina, Brazil, Chile, and Mexico) medical centers from 2008 to 2010. In this period, 5,704 gram-negative bacilli were isolated and 845 (17.7\%) were classified as Acinetobacter spp. This microorganism was responsible for $7.2 \%$ of the 6,035 bloodstream infections, $7 \%$ of the 1,442 pneumonia cases, and $9.9 \%$ of the 1,531 skin and soft tissue infections. The oxacillinases found in this study were OXA-23 and OXA-24 in Argentina, OXA-23 in Brazil, OXA-58 in Chile, and OXA-24 in Mexico $^{101}$. Figure 1 shows a map representing the description of the resistant gene OXA in the last eight years ${ }^{3,102-145}$.

\section{MOLECULAR EPIDEMIOLOGY OF A. BAUMANNII IN THE WORLD}

In France, $110 \mathrm{~A}$. baumannii clinical strains were isolated between 2010 and 2011. Of these, 90 isolates harbored $b l a_{\text {OXA-23 }}, 12$ $b l a_{\mathrm{OXA}-24}$, and $8 b l a_{\text {OXA-58 }}$. One of the isolates simultaneously displayed $b l a_{\mathrm{OXA}-23}$ and $b l a_{\text {PER-1 }}$, and 2 isolates possessed $b l a_{\text {OXA-23 }}$ and $b l a_{\text {OXA-58 }}$. Pulsed-field gel electrophoresis (PFGE) analysis showed 30 clusters and MLST revealed 11 STs (ST115, ST1, ST2, ST10, ST20, ST25, ST79, ST85, ST107, ST108, and ST125) ${ }^{43}$. A study conducted in China evaluated 57 clinical isolates of carbapenem-resistant $A$. baumannii that were positive for the $b l a_{\text {OXA-23 }} / I S A b a l$ and $b l a_{\text {OXA-51 }}$ genes, harboring ST75 and ST137 ${ }^{145}$. In addition, a Chinese hospital identified transposons Tn2006, Tn2007, and Tn2008 in 59 clinical isolates of OXA-23-producing A. baumannii ${ }^{146}$.

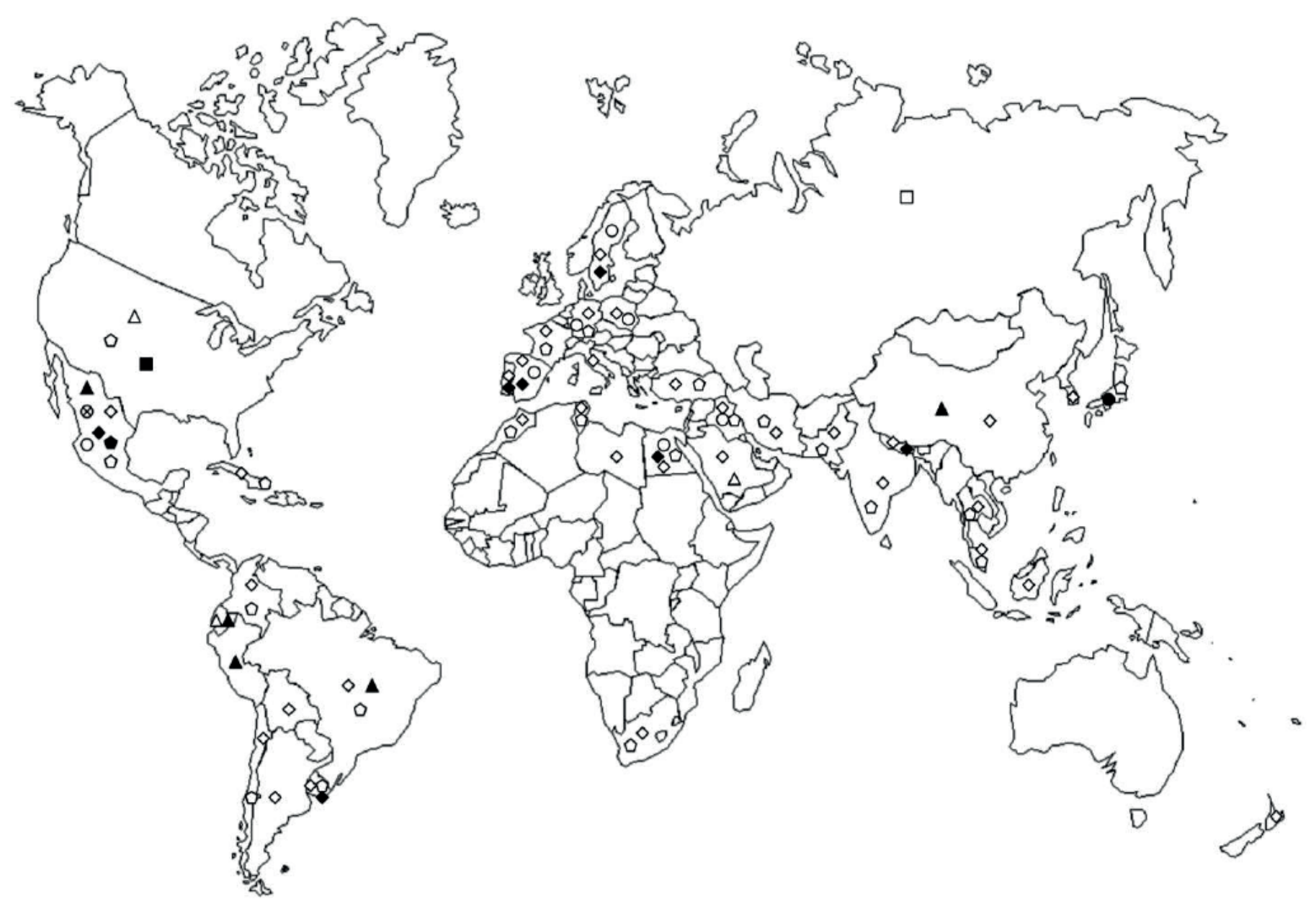

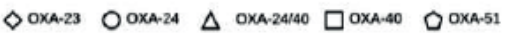


In Saudi Arabia, 107 A. baumannii clinical isolates were identified, of which 75 harbored the genes $b l a_{\text {TEM }}$ and $b l a_{\text {СТХ-M }}$ $(\mathrm{n}=86)$, bla $_{\mathrm{OXA}-51}(\mathrm{n}=100)$, and bla $\mathrm{OXA}-23(\mathrm{n}=97)$. MLST analysis identified ST195, ST557, ST208, ST499, ST218, ST231, ST222, and ST286, all belonging to CC2, except ST231 $1^{147}$. In the United States, in 2008 and 2009, 65 A. baumannii clinical isolates producing $b l a_{\text {OXA-51 }} / I S A b a l$ were found in different hospitals, harboring bla $a_{\text {OXA-23 }}(65 / 65)$ and bla $a_{\text {OXA-40 }}$ genes $(09 / 65)$. PFGE analysis indicated 24 clusters, whereas MLST identified ST1, ST2, ST77, ST79, ST123, ST124, CC1, and CC2 ${ }^{148}$. A total of 149 clinical isolates of $A$. baumannii, containing bla $_{\text {OХA-58 }}(\mathrm{n}=31)$, bla $_{\text {OХА-58 }} /$ $I S A b a 3(\mathrm{n}=14)$, and $b l a_{\mathrm{OXA}-72}(\mathrm{n}=18)$ were isolated from different hospitals in Egypt. These presented as 54 clusters by PFGE and ST763, ST777, ST369, ST762, and ST229 were identified ${ }^{149}$.

In South Africa, 94 clinical isolates of $A$. baumannii were found in different hospitals; 93 carried the $b l a_{\text {OXA-51 }}$ gene and 72 the $b l a_{\text {OXA-23 }}$. PFGE analysis grouped the isolates into 4 clusters with 5 STs (ST106, ST258, ST339, ST502, ST758, ST848), in which ST258 and ST758 corresponded to the international clone I, and ST502 and ST848 to the international clone $\mathrm{II}^{150}$. In India, 100 A. baumannii strains showed high genetic variability. MLST identified ST110, ST108, ST194, ST14, ST146, ST69, ST188, ST386, ST387, ST388, ST389, ST390, and ST391 ${ }^{151}$. A total of 160 A. baumannii clinical isolates were identified in Vietnam, of which 119 were MDR or extensively resistant, presenting a high level of resistance against third- and fourth-generation cephalosporins. Of these, 128 isolates harbored the $b l a_{\mathrm{OXA}-51}$ and $b l a_{\mathrm{OXA}-23}$ genes associated with the ISAbal element. MLST analysis identified 16 STs from 23 isolates, confirmed new STs, and some isolates belonged to ST $136^{152}$.

In Malaysia, 162 clinical isolates of MDR A. baumannii were identified, of which 128 were resistant to carbapenems. The $b l a_{\text {OXA-23, }}, b l a_{\text {OXA-IMP, }}$ and $b l a_{\text {OXA-ADC }}$ genes were identified, and ISAbal, upstream of the $b l a_{\text {OXA-23 }}$ and $b l a_{\text {OXA-ADC }}$ genes, was also found. Point mutations in gyrA (Ser83Leu) and parC (Ser80Leu), which provide resistance to ciprofloxacin, were also identified in the isolates. MLST identified two predominant STs (ST195 and ST208) ${ }^{104}$.

Molecular typing of $A$. baumannii provides a better understanding of the epidemiology of outbreaks and identification of crosstransmission, as well as assisting in the monitoring and control of nosocomial infections ${ }^{47,153}$. Thus, several methods have been used to study the molecular epidemiology of $A$. baumannii and analyze the mechanisms involved in the resistance of this microorganism.

\section{CONCLUSION}

The increase in healthcare-associated infection (HAI) rates connected to $A$. baumannii antimicrobial resistance has become a major public health challenge worldwide. A. baumannii possesses several resistance mechanisms. However, hydrolysis by OXA-type carbapenemases and metallo- $\beta$-lactamases are considered the most prevalent mechanisms conferring resistance to most beta-lactam antibiotics and reduce therapeutic options. This study highlights the occurrence of outbreaks in hospital settings, especially in ICUs, which are commonly related to prolonged hospital stays and invasive procedures. Thus, epidemiological studies are important for monitoring the occurrence of A. baumannii clinical isolates and may assist in the implementation of appropriate measures, contributing to the control of hospital infections.

\section{ACKNOWLEDGMENTS}

We are grateful to the Universidade Federal da Grande Dourados (UFGD) and the research group in Molecular Biology of Microorganisms of this institution for their support.

\section{FINANCIAL SUPPORT}

Mariana Neri Lucas Kurihara and Romário Oliveira de Sales received a scholarship from National Council for Science and Technological Development (CNPq), Késia Esther da Silva and Wirlaine Glauce Maciel from Coordenação de Aperfeiçoamento de Pessoal de Nível Superior (CAPES). The sponsors had no role in the collection, analysis and interpretation of data or the writing of the manuscript.

\section{AUTHORS' CONTRIBUTION}

WGM: Study conception and design; WGM, MNLK: Acquisition of data; MNLK, ROS, KES: Analysis and interpretation of data; MNLK, SS: Drafting of manuscript;

SS: Critical revision. Authors give final approval of the version to be submitted and any revised version.

\section{CONFLICT OF INTEREST}

The authors declare that there is no conflict of interest.

\section{REFERENCES}

1. Rossau R, Van Landschoot A, Gillis M, De Ley J. Taxonomy of Moraxellaceae fam. nov., a new bacterial family to accommodate the genera Moraxella, Acinetobacter, and Psychrobacter and related organisms. Int J Syst Bacteriol. 1991;41(2):310-29.

2. Euzeby JP. List of bacterial names with standing in nomenclature: a folder available on the internet. Int J Syst Bacteriol. 1997;47:590-2.

3. Vincent J-L, Rello J, Marshall J, Silva E, Anzueto A, Martin CD, et al. International study of the prevalence and outcomes of infection in intensive care units. JAMA. 2009;302(21):2323-9.

4. Peleg AY, Seifert H, Paterson DL. Acinetobacter baumannii: emergence of a successful pathogen. Clin Microbiol Rev. 2008;21(3):538-82.

5. Levy-Blitchtein S, Roca I, Plasencia-Rebata S, Vicente-Taboada W, Velásquez-Pomar J, Muñoz L, et al. Emergence and spread of carbapenem-resistant Acinetobacter baumannii international clones II and III in Lima, Peru article. Emerg Microbes Infect. 2018;7(1):119-28.

6. Guo H, Qin J, Xiang J. Surveillance for and susceptibility of Acinetobacter baumannii in a large hospital and burn center in Shanghai, China, 2007-2013. Am J Infect Control. 2016;44(12):1718-9.

7. Nasr P. Genetics, epidemiology, and clinical manifestations of multidrugresistant Acinetobacter baumannii. J Hosp Infect. 2020;104(1):4-11.

8. Doi Y, Murray GL, Peleg AY. Acinetobacter baumannii: Evolution of antimicrobial resistance-treatment options. Semin Respir Crit Care Med. 2015;36(1):85-98.

9. Pakharukova N, Tuittila M, Paavilainen S, Malmi H, Parilova O, Teneberg S. Structural basis for Acinetobacter baumannii biofilm formation. Proc Natl Acad Sci U S A. 2018;115(21):5558-63.

10. Lee HY, Hsu SY, Hsu JF, Chen CL, Wang YH, Chiu CH. Risk factors and molecular epidemiology of Acinetobacter baumannii bacteremia in neonates. J Microbiol Immunol Infect. 2018;51(3):367-76.

11. World Health Organization (WHO). Antibacterial Agents in preclinical development. Geneva: WHO; 2019. 20 p. 
12. Zhou H, Yao Y, Zhu B, Ren D, Yang Q, Fu Y, et al. Risk factors for acquisition and mortality of multidrug-resistant Acinetobacter baumannii bacteremia. Medicine. 2019;98(13):e14937.

13. Gramatniece A, Silamikelis I, Zahare I, Urtans V, Zahare I, Dimina E, et al. Control of Acinetobacter baumannii outbreak in the neonatal intensive care unit in Latvia: whole-genome sequencing powered investigation and closure of the ward. Antimicrob Resist Infect Control. 2019;8:84.

14. Smani Y, Fab̀rega A, Roca I, Sańchez-Encinales V, Vila J, Pachón J. Role of OmpA in the multidrug resistance phenotype of Acinetobacter baumannii. Antimicrob Agents Chemother. 2014;58(3):1806-8.

15. Zahn M, Bhamidimarri SP, Baslé A, Winterhalter M, Van den Berg B. Structural Insights into Outer Membrane Permeability of Acinetobacter baumannii. Struct. 2016;24(2):221-31.

16. Coyne S, Courvalin P, Périchon B. Efflux-mediated antibiotic resistance in Acinetobacter spp. Antimicrob Agents Chemother. 2011;55(3):947-53.

17. West AH, Stock AM. Histidine kinases and response regulator proteins in two-component signaling systems. Trends in Biochemical Sciences. 2001;26(6):369-76.

18. Wieczorek P, Sacha PHT, Zórawski M, Krawczyk M, Tryniszewska E. Multidrug resistant Acinetobacter baumannii - The role of AdeABC (RND family) efflux pump in resistance to antibiotics. Folia Histochem Cytobiol. 2008;46(3):257-67.

19. Roca I, Marti S, Espinal P, Martínez P, Gibert I, Vila J. CraA, a major facilitator superfamily efflux pump associated with chloramphenicol resistance in Acinetobacter baumannii. Antimicrob Agents Chemother. 2009;53(9):4013-4

20. Hassan KA, Jackson SM, Penesyan A, Patching SG, Tetu SG, Eijkelkamp BA, et al. Transcriptomic and biochemical analyses identify a family of chlorhexidine efflux proteins. Proc Natl Acad Sci U S A. 2013;110(50):20254-9.

21. Su XZ, Chen J, Mizushima T, Kuroda T, Tsuchiya T. AbeM, an H+coupled Acinetobacter baumannii multidrug efflux pump belonging to the MATE family of transporters. Antimicrob Agents Chemother. 2005;49(10):4362-4.

22. Pérez-Varela M, Corral J, Aranda J, Barbé J. Functional Characterization of AbaQ, a Novel Efflux Pump Mediating Quinolone Resistance in Acinetobacter baumannii. Antimicrob Agents Chemother. 2018;62(9):e00906-18.

23. Ostrer L, Khodursky RF, Johnson JR, Hiasa H, Khodursky A. Analysis of Mutational Patterns in Quinolone Resistance-Determining Regions of GyrA and ParC of Clinical Isolates. Int J Antimicrob Agents. 2018; S0924-8579(18):30366-2.

24. Zaki MES, Abou ElKheir N, Mofreh M. Molecular Study of Quinolone Resistance Determining Regions of gyrA Gene and parC Genes in Clinical Isolates of Acinetobacter baumannii Resistant to Fluoroquinolone. Open Microbiol J. 2018;12:116-22.

25. Papp-Wallace KM, Senkfor B, Gatta J, Chai W, Taracila MA, Shanmugasundaram $\mathrm{V}$, et al. Early Insights into the Interactions of Different $\beta$-Lactam Antibiotics and $\beta$-Lactamase Inhibitors against Soluble Forms of Acinetobacter baumannii PBP1a and Acinetobacter sp. PBP3. Antimicrob Agents Chemother. 2012;56(11):5687-92.

26. Sauvage E, Kerff F, Terrak M, Ayala JA, Charlier P. The penicillinbinding proteins: structure and role in peptidoglycan biosynthesis. FEMS Microbiol Rev. 2008;32(2):234-58.

27. Cayô R, Rodríguez M-C, Espinal P, Fernández-Cuenca F, Ocampo-Sosa AA, Pascual A, et al. Analysis of Genes Encoding Penicillin-Binding Proteins in Clinical Isolates of Acinetobacter baumannii. Antimicrob Agents Chemother. 2011;55(12),5907-13.
28. Fernandez-Cuenca F, Martínez-Martínez L, Conejo MC, Ayala JA, Perea EJ, Pascual A. Relationship between beta-lactamase production, outer membrane protein and penicillin-binding protein profiles on the activity of carbapenems against clinical isolates of Acinetobacter baumannii. J Antimicrob Chemother. 2003;51(3),565-74.

29. Hawkey J, Ascher DB, Judd LM, Wick RR, Kostoulias X, Cleland H, et al. Evolution of carbapenem resistance in Acinetobacter baumannii during a prolonged infection. Microb.Genomics. 2018;4: e000165.

30. Boll JM, Crofts AA, Peters K, Cattoir V, Vollmer W, Davies BW, et al. A penicillin-binding protein inhibits selection of colistin-resistant, lipooligosaccharide-deficient Acinetobacter baumannii. Proc Natl Acad Sci U S A. 2016;113(41):E6228-37.

31. Moffatt JH, Harper M, Boyce JD. Mechanisms of Polymyxin Resistance. In J. Li, R. L. Nation, K. S. Kaye (Eds.), Polymyxin Antibiotics: From Laboratory Bench to Bedside (1st ed., 2019. pp. 55-71). (Advances in Experimental Medicine and Biology; Vol. 1145). Springer.

32. Garnacho-Montero J, Timsit J-F. Managing Acinetobacter baumannii infections. Curr Opin Infect Dis 2018,32:69-76.

33. Dortet L, Potron A, Bonnin RA, Plesiat P, Naas T, Filloux A, et al. Rapid detection of colistin resistance in Acinetobacter baumannii using MALDITOF-based lipidomics on intact bacteria. Scientific Reports. 2018;8(1).

34. Beceiro A, Llobet E, Aranda J, Bengoechea JA, Doumith M, Hornsey $\mathrm{M}$, et al. Phosphoethanolamine modification of lipid A in colistinresistant variants of Acinetobacter baumannii mediated by the pmrAB two-component regulatory system. Antimicrob Agents Chemother. 2011;55(7):3370-9.

35. Rawat D, Nair D. Extended-spectrum $\beta$-lactamases in Gram Negative Bacteria. J Glob Infect Dis. 2010;2(3):263-74.

36. Da Silva GJ, Domingues S. Interplay between Colistin Resistance, Virulence and Fitness in Acinetobacter baumannii. Antibiot. 2017;6,28.

37. Hameed F, Khan MA, Muhammad H, Sarwar T, Bilal H, Rehman TU. Plasmid-mediated mcr-1 gene in Acinetobacter baumannii and Pseudomonas aeruginosa: first report from Pakistan. Rev Soc Bras Med Trop. 2019;52:e20190237.

38. Queenan AM, Bush K. Carbapenemases: The versatile $\beta$-lactamases. Clin Microbiol Rev. 2007;20(3):440-58.

39. Robledo IE, Aquino EE, Santé MI, Santana JL, Otero DM, Léon CF, et al. Detection of KPC in Acinetobacter spp. in Puerto Rico. Antimicrob Agents Chemother. 2010;54(3):1354-7.

40. Bogaerts P, Naas T, El Garch F, Cuzon G, Deplano A, Delaire T, et al. GES extended-spectrum $\beta$-lactamases in Acinetobacter baumannii isolates in Belgium. Antimicrob Agents Chemother. 2010;54(11):4872-8.

41. Bush K, Jacoby GA, Medeiros AA. A functional classification scheme for $\beta$-lactamases and its correlation with molecular structure. Antimicrob Agents Chemother. 1995;39(6):1211-33.

42. Poirel L, Nordmann P. Carbapenem resistance in Acinetobacter baumannii: Mechanisms and epidemiology. Clin Microbiol Infect. 2006;12(9):826-36.

43. Jeannot K, Diancourt L, Vaux S, Thouverez M, Ribeiro A, Coignard $\mathrm{B}$, et al. Molecular epidemiology of carbapenem non-susceptible Acinetobacter baumannii in France. PLoS One. 2014;9(12):e115452.

44. Bush K, Jacoby GA. Updated functional classification of betalactamases. Antimicrob Agents Chemother. 2010;54(3):969-76.

45. Karah N, Jolley KA, Hall RM, Uhlin BE. Database for the ampC alleles in Acinetobacter baumannii. PLoS One. 2017;12(5):e0176695.

46. Ning NZ, Liu X, Bao CM, Chen SM, Cui EB, Zhang JL, et al. Molecular epidemiology of bla OXA-23-producing carbapenem-resistant 
Acinetobacter baumannii in a single institution over a 65 -month period in north China. BMC Infect Dis. 2017;17(1):14.

47. Kamolvit W, Sidjabat HE, Paterson DL. Molecular Epidemiology and Mechanisms of Carbapenem Resistance of Acinetobacter spp. in Asia and Oceania. Microb Drug Resist. 2015;21(4):424-34.

48. Wang D, Yan D, Hou W, Zeng X, Qi Y, Chen J. Characterization of blaOxA-23 gene regions in isolates of Acinetobacter baumannii. J Microbiol Immunol Infect. 2015;48(3):284-90.

49. Martínez P, Mattar S. Imipenem-resistant Acinetobacter baumannii carrying the ISABA1-BLAOXA-23, 51 and ISABA1-BLAADC-7 genes in Monteria, Colombia. Brazilian J Microbiol. 2012;43(4):1274-80.

50. Bakour S, Alsharapy SA, Touati A, Rolain J-M. Characterization of Acinetobacter baumannii clinical isolates carrying bla(OXA-23) carbapenemase and 16S rRNA methylase armA genes in Yemen. Microb Drug Resist. 2014;20(6):604-9.

51. Polotto M, Casella T, Tolentino FM, Mataruco MM, Porto NKM, Binhardi $\mathrm{MFB}$, et al. Investigation of carbapenemases and aminoglycoside modifying enzymes of Acinetobacter baumannii isolates recovered from patients admitted to intensive care units in a tertiary-care hospital in Brazil. Rev Soc Bras Med Trop. 2020;53:e20190044.

52. Magnet S, Blanchard JS. Molecular insights into aminoglycoside action and resistance. Chem Rev. 2005;105:477-98.

53. Hong SB, Shin KS, Ha J, Han K. Co-existence of blaOXA-23 and armA in multidrug-resistant Acinetobacter baumannii isolated from a hospital in South Korea. J Med Microb. 2013;62(Pt_6):836-44.

54. Cho YJ, Moon DC, Jin JS, Choi CH, Lee YC, Lee JC. Genetic basis of resistance to aminoglycosides in Acinetobacter spp. and spread of armA in Acinetobacter baumannii sequence group 1 in Korean hospitals. Diagn Microb Infect Dis. 2009;64(2),185-90.

55. Kobs VC, Ferreira JA, Bobrowicz TA, Ferreira LE, Deglmann RC, Westphal GA, et al. The role of the genetic elements blaoxa and ISAbal in the Acinetobacter calcoaceticus-Acinetobacter baumannii complex in carbapenem resistance in the hospital setting. Rev Soc Bras Med Trop. 2016;49(4):433-40.

56. Héritier C, Poirel L, Lambert T, Nordmann P. Contribution of acquired carbapenem-hydrolyzing oxacillinases to carbapenem resistance in Acinetobacter baumannii. Antimicrob Agents Chemother. 2005;49(8):3198-202.

57. Hamidian M, Ambrose SJ, Hall RM. A large conjugative Acinetobacter baumannii plasmid carrying the sul2 sulphonamide and strAB streptomycin resistance genes. Plasmid. 2016;87-88:43-50.

58. Hamidian M, Hall RM. pACICU2 is a conjugative plasmid of Acinetobacter carrying the aminoglycoside resistance transposon TnaphA6. J Antimicrob Chemother. 2014;69(4):1146-8.

59. Nigro S, Hall RM. Distribution of the blaOXA-23-containing transposons Tn2006 and Tn2008 in Australian carbapenem-resistant Acinetobacter baumannii isolates. J Antimicrob Chemother. 2015;70(8):2409-11.

60. Zong Z, Zhang X. BlaNDM-1-carrying Acinetobacter johnsonii detected in hospital sewage. J Antimicrob Chemother. 2013;68(5):1007-10.

61. Najar Peerayeh S, Karmostaji A. Molecular Identification of Resistance Determinants, Integrons and Genetic Relatedness of Extensively Drug Resistant Acinetobacter baumannii Isolated From Hospitals in Tehran, Iran. Jundishapur J Microbiol. 2015;8(7):e27021.

62. Azizi O, Shakibaie MR, Badmasti F, Modarresi F, Ramazanzadeh R, Mansouri S, et al. Class 1 integrons in non-clonal multidrugresistant Acinetobacter baumannii from Iran, description of the new blaIMP-55 allele in in1243. J Med Microbiol. 2016;65(9):928-36.

63. Khorsi K, Messai Y, Hamidi M, Ammari H, Bakour R. High prevalence of multidrug-resistance in Acinetobacter baumannii and dissemination of carbapenemase-encoding genes blaOXA-23-like, blaOXA-24like and blaNDM-1 in Algiers hospitals. Asian Pac J Trop Med. 2015;8(6):438-46

64. Mugnier PD, Poirel L, Naas T, Nordmann P. Worldwide dissemination of the blaOXA-23 Carbapenemase gene of Acinetobacter baumannii. Emerg Infect Dis. 2010;16(1):35-40.

65. De Figueiredo DQ, Castro LFS, Santos KRN, Teixeira LM, De Mondlno SSB. Detecção de metalo-beta-lactamases em amostras hospitalares de Pseudomonas aeruginosa e Acinetobacter baumannii. J Bras Patol Med Lab. 2009;45(3):177-84.

66. Lucas DD, Crane B, Wright A, Han ML, Moffatt J, Bulach D, et al. Emergence of high-level colistin resistance in an Acinetobacter baumannii clinical isolate mediated by inactivation of the global regulator H-NS. Antimicrob Agents Chemother. 2018; 62(7). pii:e02442-17.

67. Henig O, Weber G, Hoshen MB, Paul M, German L, Neuberger A, et al. Risk factors for and impact of carbapenem-resistant Acinetobacter baumannii colonization and infection: matched case-control study. Eur J Clin Microbiol Infect Dis. 2015;34(10):2063-8.

68. Chusri S, Silpapojakul K, McNeil E, Singkhamanan K, Chongsuvivatwong V. Impact of antibiotic exposure on occurrence of nosocomial carbapenem-resistant Acinetobacter baumannii infection: a case control study. J Infect Chemother. 2015;21(2):90-5.

69. Rocha LDA Da, Vilela CAP, Cezário RC, Almeida AB, Gontijo Filho P. Ventilator-associated pneumonia in an adult clinical-surgical intensive care unit of a Brazilian university hospital: incidence, risk factors, etiology, and antibiotic resistance. Braz J Infect Dis. 2008;12(1):80-5.

70. Brotfain E, Borer A, Koyfman L, Saidel-Odes L, Frenkel A, Gruenbaum SE, et al. Multidrug Resistance Acinetobacter Bacteremia Secondary to Ventilator-Associated Pneumonia: Risk Factors and Outcome. J Intensive Care Med. 2017;32(9):528-34

71. Ellis D, Cohen B, Liu J, Larson E. Risk factors for hospital-acquired antimicrobial-resistant infection caused by Acinetobacter baumannii. Antimicrob Resist Infect Control. 2015;4:40.

72. Moghnieh R, Siblani L, Ghadban D, El Mchad H, Zeineddine R, Abdallah D, et al. Extensively drug-resistant Acinetobacter baumannii in a Lebanese intensive care unit: risk factors for acquisition and determination of a colonization score. J Hosp Infect. 2016;92(1):47-53.

73. Blanco N, Harris AD, Rock C, Johnson JK, Pineles L, Bonomo RA, et al. Risk factors and outcomes associated with multidrug- resistant Acinetobacter baumannii upon intensive care unit admission. Antimicrob Agents Chemother. 2018;62(1):e01631-17.

74. Guo N, Xue W, Tang D, Ding J, Zhao B. Risk factors and outcomes of hospitalized patients with blood infections caused by multidrugresistant Acinetobacter baumannii complex in a hospital of Northern China. Am J Infect Control. 2016;44(4):e37-9.

75. Nutman A, Glick R, Temkin E, Hoshen M, Edgar R, Braun T, et al. A case-control study to identify predictors of 14-day mortality following carbapenem-resistant Acinetobacter baumannii bacteraemia. Clin Microbiol Infect. 2014;20(12):1028-34.

76. Romanellia RMC, Anchieta LM, Mourão MVA, Campos FA, Loyola FC, Mourão PHO, et al. Risk factors and lethality of laboratory-confirmed bloodstream infection caused by non-skin contaminant pathogens in neonates. J Pediatr (Rio J). 2013; 89(2):189-96.

77. Wei H-M, Hsu Y-L, Lin H-C, Hsieh T-H, Yen T-Y, Lin H-C, et al. Multidrug-resistant Acinetobacter baumannii infection among neonates in a neonatal intensive care unit at a medical center in central Taiwan. J Micro Immun Infect. 2014; 48(5):531-9.

78. Hosoglu S, Hascuhadar M, Yasar E, Uslu S, Aldudak B. Control of an Acinetobacter baumannii outbreak in a neonatal ICU without 
suspension of service: A devastating outbreak in Diyarbakir, Turkey. Infection. 2012;40(1):11-8.

79. Zarrilli R, Di Popolo A, Bagattini M, Giannouli M, Martino D, Barchitta $\mathrm{M}$, et al. Clonal spread and patient risk factors for acquisition of extensively drug-resistant Acinetobacter baumannii in a neonatal intensive care unit in Italy. J Hosp Infect. 2012;82(4):260-5.

80. De Oliveira CP, Atta EH, Da Silva ARA. Infection with multidrugresistant gram-negative bacteria in a pediatric oncology intensive care unit: Risk factors and outcomes. J Pediatr (Rio J). 2015;91(5):435-41.

81. Deng C, Li X, Zou Y, Wang J, Wang J, Namba F, et al. Risk factors and pathogen profile of ventilator-associated pneumonia in a neonatal intensive care unit in China. Pediatr Int. 2011;53(3):332-7.

82. Reddy D, Morrow BM, Argent AC. Acinetobacter baumannii infections in a South African paediatric intensive care unit. J Trop Pediatr. 2015; 61(3):182-7.

83. Kumar A, Randhawa VS, Nirupam N, Rai Y, Saili A. Risk factors for carbapenem-resistant Acinetobacter baumanii blood stream infections in a neonatal intensive care unit, Delhi, India. J Infect Dev Ctries. 2014;8(8):1049-54.

84. Thatrimontrichai A, Apisarnthanarak A, Chanvitan P, Janjindamai W, Dissaneevate S, Maneenil G. Risk factors and outcomes of carbapenemresistant Acinetobacter baumannii bacteremia in neonatal intensive care unit: a case-case-control study. Pediatr Infect Dis J. 2013;32(2):140-5.

85. Tran HT, Doyle LW, Lee KJ, Dang NM, Graham SM. A high burden of late-onset sepsis among newborns admitted to the largest neonatal unit in central Vietnam. J Perinatol. 2015;35(10):846-51.

86. Hsu JF, Chu SM, Lien R, Chiu CH, Chiang MC, Fu RH, et al. Casecontrol analysis of endemic Acinetobacter baumannii bacteremia in the neonatal intensive care unit. Am J Infect Control. 2014;42(1):23-7.

87. Punpanich W, Nithitamsakun N, Treeratweeraphong V, Suntarattiwong P. Risk factors for carbapenem non-susceptibility and mortality in Acinetobacter baumannii bacteremia in children. Int $\mathrm{J}$ Infect Dis. 2012;16(11):811-5.

88. Maciel WG, da Silva KE, Croda J, Cayô R, Ramos AC, de Sales RO, et al. Clonal spread of carbapenem-resistant Acinetobacter baumannii in a neonatal intensive care unit. J Hosp Infect. 2017;98(3):300-4.

89. Kritsotakis EI, Kozhageldiyeva AG. A systematic review of the global seasonality of infections caused by Acinetobacter species in hospitalized patients. Clin Microb Infect. 2019;26(5):553-62.

90. Williams VR, Callery S, Vearncombe M, Simor AE. The role of colonization pressure in nosocomial transmission of methicillinresistant Staphylococcus aureus. Am J Infect Control. 2009;37(2),10610.

91. Castelo-Branco FCM, Moreira FF, da Paz LG. Colonization pressure and risk factors for acquisition of imipenem-resistant Acinetobacter baumannii in a medical surgical intensive care unit in Brazil. Am J Infect Control. 2013;41(3):263-5.

92. Dalia-Costa LM, Coelho JM, Souza HAPHM, Castro MES, Stier CJN, Bragagnolo KL, et al. Outbreak of carbapenem-resistant Acinetobacter baumannii producing the OXA-23 enzyme in Curitiba, Brazil. J Clin Microbiol. 2003;41(7):3403-6.

93. Carvalho KR, Carvalho-Assef APDA, Peirano G, Santos LCG dos, Pereira MJF, Asensi MD. Dissemination of multidrug-resistant Acinetobacter baumannii genotypes carrying blaOXA-23 collected from hospitals in Rio de Janeiro, Brazil. Int J Antimicrob Agents. 2009;34(1):25-8.

94. Castilho SRA, Godoy CSDM, Guilarde AO, Cardoso JL, André MCP, Junqueira-Kipnis AP, et al. Acinetobacter baumannii strains isolated from patients in intensive care units in Goiânia, Brazil: Molecular and drug susceptibility profiles. PLoS One. 2017;12(5):e0176790.

95. de Souza Gusatti C, Bertholdo LM, Otton LM, Marchetti DP, Ferreira AE, Corção G. First occurrence of bla OXA-58 in Acinetobacter baumannii isolated from a clinical sample in Southern Brazil. Braz J Microbiol. 2012;43(1):243-6.

96. Martins N, Martins IS, de Freitas WV, de Matos JA, Girão VBDC, Coelho-Souza $\mathrm{T}$, et al. Imported and Intensive Care Unit-Born Acinetobacter baumannii Clonal Complexes: One-Year Prospective Cohort Study in Intensive Care Patients. Microb Drug Resist. 2013;19(3):216-23.

97. Leal NC, Campos TL, Rezende AM, Docena C, Mendes-Marques CL, de Sá Cavalcanti FL, et al. Comparative Genomics of Acinetobacter baumannii Clinical Strains From Brazil Reveals Polyclonal Dissemination and Selective Exchange of Mobile Genetic Elements Associated With Resistance Genes. Front Microbiol. 2020;11:1176.

98. Rodríguez CH, Balderrama Yarhui N, Nastro M, Nuñez Quezada T, Castro Cañarte G, Ventura RM, et al. Molecular epidemiology of carbapenem-resistant Acinetobacter baumannii in South America. J Med Microbiol. 2016;65(10):1088-91.

99. MLST, Pasteur. Acinetobacter baumannii, MLST (Pasteur) database; 2016 [updated 2016 June 06; cited 2020 Oct 15]. Available from: https:// pubmlst.org/bigsdb?db=pubmlst_abaumannii_pasteur_seqdef

100.Chagas TPG, Carvalho KR, de Oliveira Santos IC, Carvalho-Assef APDA, Asensi MD. Characterization of carbapenem-resistant Acinetobacter baumannii in Brazil (2008-2011): Countrywide spread of OXA-23producing clones $(\mathrm{CC} 15$ and $\mathrm{CC} 79)$. Diagn Microbiol Infect Dis. 2014;79(4):468-72.

101. Gales AC, Castanheira M, Jones RN, Sader HS. Antimicrobial resistance among Gram-negative bacilli isolated from Latin America: Results from SENTRY Antimicrobial Surveillance Program (Latin America, 2008-2010). Diagn Microbiol Infect Dis. 2012;73(4):354-60.

102.Almaghrabi MK, Joseph MRP, Assiry MM, Hamid ME. MultidrugResistant Acinetobacter baumannii: An Emerging Health Threat in Aseer Region, Kingdom of Saudi Arabia. Can J Infect Dis Med Microbiol. 2018;2018:1-4.

103. Teo J, Lim TP, Hsu LY, Tan TY, Sasikala S, Hon PY, et al. Extensively drug-resistant Acinetobacter baumannii in a Thai hospital: A molecular epidemiologic analysis and identification of bactericidal Polymyxin B-based combinations. Antimicrob Resist Infect Control. 2015;4(1):2.

104.Biglari S, Hanafiah A, Mohd Puzi S, Ramli R, Rahman M, Lopes BS. Antimicrobial Resistance Mechanisms and Genetic Diversity of MultidrugResistant Acinetobacter baumannii Isolated from a Teaching Hospital in Malaysia. Microb Drug Resist. 2017;23(5):545-55.

105. Camargo CH, Tiba MR, Saes MR, De Vasconcellos FM, Dos Santos LF, Romero EC, et al. Population structure analysis of carbapenemresistant Acinetobacter baumannii clinical isolates from Brazil reveals predominance of clonal complexes 1, 15, and 79. Antimicrob Agents Chemother. 2016;60(4):2545-7.

106.Royer S, Amaral de Campos P, Araújo BF, Ferreira ML, Gonçalves IR, William da Fonseca Batistão D, et al. Molecular characterization and clonal dynamics of nosocomial blaOXA-23producing XDR Acinetobacter baumannii. PLoS One. 2018;13(6):e0198643.

107. Asai S, Umezawa K, Iwashita H, Ohshima T, Ohashi M, Sasaki M, et al. An outbreak of blaOXA-51-like- and blaOXA-66- positive Acinetobacter baumannii ST208 in the emergency intensive care unit. J Med Microbiol. 2014;63(Pt 11):1517-23.

108.Correa A, Del Campo R, Escandón-Vargas K, Perenguez M, RodríguezBanõs M, Hernández-Gómez C, et al. Distinct Genetic Diversity of 
Carbapenem-Resistant Acinetobacter baumannii from Colombian Hospitals. Microb Drug Resist. 2018;24(1):48-54.

109.Uzunoglu E, Direkel S, Kocbiyik M, Uludag SK, Cicek AC. Co-existance of isaba1/blaoxa-51/23 is increasing in carbapenem rersistant Acinetobacter baumannii isolates in Turkey. Acta Medica Mediterr. 2017;33(6):1001.

110. Jaidane N, Naas T, Mansour W, Radhia B Ben, Jerbi S, Boujaafar N, et al. Genomic analysis of in vivo acquired resistance to colistin and rifampicin in Acinetobacter baumannii. Int J Antimicrob Agents. 2018;51(2):266-9.

111. El Kettani A, Maaloum F, Diawara I, Katfy K, Harrar N, Zerouali K, et al. Prevalence of Acinetobacter baumannii bacteremia in intensive care units of ibn rochd university hospital, Casablanca. Iran J Microbiol. 2017;9(6):318-23.

112.Dahdouh E, Gómez-Gil R, Pacho S, Mingorance J, Daoud Z, Suárez M. Clonality, virulence determinants, and profiles of resistance of clinical Acinetobacter baumannii isolates obtained from a Spanish hospital. PLoS One. 2017;12(4):e0176824.

113. Lowe M, Ehlers MM, Ismail F, Peirano G, Becker PJ, Pitout JDD, et al. Acinetobacter baumannii: Epidemiological and beta-lactamase data from two tertiary academic hospitals in Tshwane, South Africa. Front Microbiol. 2018;9:1280.

114. Mathlouthi N, Ben lamine Y, Somai R, Bouhalila-Besbes S, Bakour S, Rolain J-M, et al. Incidence of OXA-23 and OXA-58 Carbapenemases Coexpressed in Clinical Isolates of Acinetobacter baumannii in Tunisia. Microb Drug Resist. 2017;24(2):136-41.

115. Chmielarczyk A, Pilarczyk-Żurek M, Kamińska W, Pobiega M, Romaniszyn D, Ziółkowski G, et al. Molecular Epidemiology and Drug Resistance of Acinetobacter baumannii Isolated from Hospitals in Southern Poland: ICU as a Risk Factor for XDR Strains. Microb Drug Resist. 2016;22(4):328-35.

116. KatchanovJ,AsarL,KluppEM,BothA, RotheC, KönigC, etal.Carbapenemresistant Gram-negative pathogens in a German university medical center: Prevalence, clinical implications and the role of novel $\beta$-lactam $/ \beta$-lactamase inhibitor combinations. PLoS One. 2018;13(4):e0195757.

117. Hujer AM, Higgins PG, Rudin SD, Buser GL, Marshall SH, Xanthopoulou $\mathrm{K}$, et al. Nosocomial outbreak of extensively drug-resistant Acinetobacter baumannii isolates containing blaOXA-237 carried on a plasmid. Antimicrob Agents Chemother. 2017; 61(11).

118. Sheck EA, Edelstein MV, Sukhorukova MV, Ivanchik NV, Skleenova EY, Dekhnich A V, et al. Epidemiology and genetic diversity of colistin nonsusceptible nosocomial Acinetobacter baumannii strains from Russia for 2013-2014. Can J Infect Dis Med Microbiol. 2017;2017:1839190.

119. Karah N, Giske CG, Sundsfjord A, Samuelsen Ø. A Diversity of OXACarbapenemases and Class 1 Integrons Among Carbapenem-Resistant Acinetobacter baumannii Clinical Isolates from Sweden Belonging to Different International Clonal Lineages. Microb Drug Resist. 2011;17(4):545-9.

120. Grosso F, Quinteira S, Peixe L. Understanding the dynamics of imipenemresistant Acinetobacter baumannii lineages within Portugal. Clin Microbiol Infect. 2011;17(8):1275-9.

121. Banerjee T, Mishra A, Das A, Sharma S, Barman H, Yadav G. High Prevalence and Endemicity of Multidrug Resistant Acinetobacter spp. in Intensive Care Unit of a Tertiary Care Hospital, Varanasi, India. J Pathog. 2018;2018:9129083.

122.Creighton J, Heffernan H, Howard J. Isolation of seven distinct carbapenemase-producing Gram-negative organisms from a single patient. J Antimicrob Chemother. 2017;72(1):317-19.

123.Handal R, Qunibi L, Sahouri I, Juhari M, Dawodi R, Marzouqa H, et al. Characterization of Carbapenem-Resistant Acinetobacter baumannii Strains Isolated from Hospitalized Patients in Palestine. Int J Microbiol. 2017;2017:8012104
124.Abdulzahra AT, Khalil MAF, Elkhatib WF. First report of colistin resistance among carbapenem-resistant Acinetobacter baumannii isolates recovered from hospitalized patients in Egypt. New Microbes New Infect. 2018;26:53-8.

125.Quiñones D, Carvajal I, Perez Y, Hart M, Perez J, Garcia S, et al. High prevalence of blaOXA-23in Acinetobacter spp. and detection of blaNDM1in A.soli in Cuba: Report from National Surveillance Program (20102012). New Microbes New Infect. 2015;7:52-6.

126.Rodríguez CH, Nastro M, Fiorilli G, Dabos L, Calvo JL, Fariña ME, et al. Trends in the resistance profiles of Acinetobacter baumannii endemic clones in a university hospital of Argentina. J Chemother. 2016;28(1):25-7.

127. Opazo A, Bello H, Domínguez M, Lima CA, Opazo A, González-Rocha G, et al. First report of blaOXA-23in Acinetobacter baumannii isolates from Chilean hospitals. J Glob Antimicrob Resist. 2015;3(1):54-5.

128.Nuñez Quezada T, Rodríguez CH, Castro Cañarte G, Nastro M, Balderrama Yarhui N, Dabos L, et al. Outbreak of bla OXA-72-producing Acinetobacter baumannii in South America. J Chemother. 2017;29(5):321-4.

129. Saharman YR, Karuniawati A, Sedono R, Aditianingsih D, Sudarmono P, Goessens WH, et al. Endemic carbapenem-nonsusceptible Acinetobacter baumannii-calcoaceticus complex in intensive care units of the national referral hospital in Jakarta, Indonesia. Antimicrob Resist Infect Control. 2018;7(1):5.

130.Cerezales M, Ocampo-Sosa AA, Montes LÁ, Ríos CD, Bustamante Z, Santos J, et al. High prevalence of extensively drug-resistant Acinetobacter baumannii at a children hospital in Bolivia. Pediatr Infect Dis J. 2018;37(11):1118-23.

131. Chen Y, Yang Y, Liu L, Qiu G, Han X, Tian S, et al. High prevalence and clonal dissemination of OXA-72-producing Acinetobacter baumannii in a Chinese hospital: a cross sectional study. BMC Infect Dis. 2018;18(1):491.

132.Shirmohammadlou N, Zeighami H, Haghi F, Kashefieh M. Resistance pattern and distribution of carbapenemase and antiseptic resistance genes among multidrug-resistant Acinetobacter baumannii isolated from intensive care unit patients. J Med Microbiol. 2018;67(10):1467-73.

133. Bado I, Papa-Ezdra R, Delgado-Blas JF, Gaudio M, Gutiérrez C, Cordeiro NF, et al. Molecular characterization of carbapenem-resistant Acinetobacter baumannii in the intensive care unit of Uruguay's University Hospital identifies the first rmtC gene in the species. Microb Drug Resist. 2018;24(7):1012-9.

134.Al-Agamy MH, Khalaf NG, Tawfick MM, Shibl AM, El Kholy A. Molecular characterization of carbapenem-insensitive Acinetobacter baumannii in Egypt. Int J Infect Dis. 2014;22:49-54.

135. Kazi M, Nikam C, Shetty A, Rodrigues C. Dual-tubed multiplex-PCR for molecularcharacterization of carbapenemases isolated among Acinetobacter spp. and Pseudomonas spp. J Appl Microbiol. 2015;118(5):1096-102.

136.Hasan B, Perveen K, Olsen B, Zahra R. Emergence of carbapenem-resistant Acinetobacter baumannii in hospitals in Pakistan. J Med Microbiol. 2014;63(1):50-5.

137. Irfan S, Turton JF, Mehraj J, Siddiqui SZ, Haider S, Zafar A, et al. Molecular and epidemiological characterisation of clinical isolates of carbapenemresistant Acinetobacter baumannii from public and private sector intensive care units in Karachi, Pakistan. J Hosp Infect. 2011;78(2):143-8.

138. Shrestha S, Tada T, Miyoshi-Akiyama T, Ohara H, Shimada K, Satou K, et al. Molecular epidemiology of multidrug-resistant Acinetobacter baumannii isolates in a university hospital in Nepal reveals the emergence of a novel epidemic clonal lineage. Int J Antimicrob Agents. 2015;46(5):526-31.

139.Jamal S, Al Atrouni A, Rafei R, Dabboussi F, Hamze M, Osman M. Molecular mechanisms of antimicrobial resistance in Acinetobacter baumannii, with a special focus on its epidemiology in Lebanon. J Glob Antimicrob Resist. 2018;15:154-63. 
140.Jung JY, Park MS, Kim SE, Park BH, Son JY, Kim EY, et al. Risk factors for multi-drug resistant Acinetobacter baumannii bacteremia in patients with colonization in the intensive care unit. BMC Infect Dis. 2010;10:228.

141. Jang TN, Lee SH, Huang CH, Lee CL, Chen WY. Risk factors and impact of nosocomial Acinetobacter baumannii bloodstream infections in the adult intensive care unit: a case-control study. J Hosp Infect. 2009;73(2):143-50.

142. Ye JJ, Huang CT, Shie SS, Huang PY, Su LH, Chiu CH. Multidrug resistant Acinetobacter baumannii: Risk factors for appearance of imipenem resistant strains on patients formerly with susceptible strains. PLOS ONE. 2010;5(4):e9947.

143. Pagano M, Barin J, Martins AF, Zavascki AP. High Endemic Rates of OXA23-Producing Carbapenem-Resistant Acinetobacter baumannii Isolates Caused by the Persistence of Major Clones in Hospitals in a Brazilian City 5 Years After an Outbreak. Infect Control Hosp Epidemiol. 2015; 36(7): 860-2.

144.Brito DVD, Brito CS de, Resende DS, Moreira do Ó J, Abdallah VOS, Gontijo Filho PP. Nosocomial infections in a Brazilian neonatal intensive care unit: a 4-year surveillance study. Rev Soc Bras Med Trop. 2010; 43(6):633-7.

145. Takagi, EH, Lincopan, N, Cassettari, VC, Passadore, LF, Mamizuka, EM, Martinez, MB. Carbapenem-resistant Acinetobacter baumannii outbreak at university hospital. Braz J Microbiol. 2009;40(2),339-41.

146.Dai W, Huang S, Sun S, Cao J, Zhang L. Nosocomial spread of carbapenemresistant Acinetobacter baumannii (types ST75 and ST137) carrying blaOXA-23-like gene with an upstream ISAba1 in a Chinese hospital. Infect Genet Evol. 2013;14:98-101.
147. Alyamani EJ, Khiyami MA, Booq RY, Alnafjan BM, Altammami MA, Bahwerth FS. Molecular characterization of extended-spectrum betalactamases (ESBLs) produced by clinical isolates of Acinetobacter baumannii in Saudi Arabia. Ann Clin Microbiol Antimicrob. 2015;14(1):38.

148. Adams-Haduch JM, Onuoha EO, Bogdanovich T, Tian GB, Marschall J, Urban CM, et al. Molecular epidemiology of carbapenem-nonsusceptible Acinetobacter baumannii in the United States. J Clin Microbiol. 2011;49(11):3849-54.

149. Bocanegra-Ibarias P, Pena-López C, Camacho-Ortiz A, Llaca-Díaz J, Silva-Sánchez J, Barrios H, et al. Genetic characterisation of drug resistance and clonal dynamics of Acinetobacter baumannii in a hospital setting in Mexico. Int J Antimicrob Agents. 2015;45(3):309-13.

150.Lowings M, Ehlers MM, Dreyer AW, Kock MM. High prevalence of oxacillinases in clinical multidrug-resistant Acinetobacter baumannii isolates from the Tshwane region, South Africa-an update. BMC Infect Dis. 2015;15(1):521.

151. Rynga D, Shariff M, Deb M. Phenotypic and molecular characterization of clinical isolates of Acinetobacter baumannii isolated from Delhi, India. Ann Clin Microbiol Antimicrob. 2015;14(1):40.

152.Anh NT, Nga TV, Tuan HM, Tuan NS, Chau NV, Baker S, et al. Molecular epidemiology and antimicrobial resistance phenotypes of Acinetobacter baumannii isolated from patients in three hospitals in southern Vietnam. J Med Microbiol. 2017;66(1):46-53.

153.Al-Hamad A, Pal T, Leskafi H, Abbas H, Hejles H, Alsubikhy F, et al. Molecular characterization of clinical and environmental carbapenem resistant Acinetobacter baumannii isolates in a hospital of the Eastern Region of Saudi Arabia. J Infect Public Health. 2020;13(4)632-6. 